Recepción: 20 / 04 / 2017

Aceptación: 20 / 05 / 2017

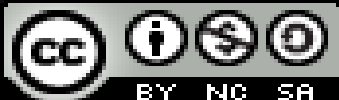

Ciencias Médicas

Publicación: 15 / 07 / 2017

Artículo Científico

\title{
Situación materna VIH como factor predisponente de vitalidad neonatal
}

\author{
Maternal HIV status as a predisposing factor \\ of neonatal vitality
}

\section{Estado materna HIV como um factor de predisposição vitalidade neonatal}

Filipo P. Montece-López ${ }^{\text {I }}$ filipoaa@hotmail.com

Marlene I. Saltos-Calvache II marle.saltosc@ug.edu.ec

Carlota M. Palma-Estrada III carlota.palmae@ug.edu.ec

Julio De la Torre-Chávez IV julio.delatorrec@ug.edu.ec

Correspondencia: filipoaa@hotmail.com

\footnotetext{
Especialista Neonatología y Terapia Intensiva Neonatal; Especialista en Pediatría; Doctor en Medicina y Cirugía; Hospital General Guasmo Sur, Universidad de Guayaquil, Guayaquil, Ecuador.

Magister en Gerencia Hospitalaria; Medico; Universidad de Guayaquil, Guayaquil, Ecuador.

III. Magister en Nutrición; Diplomado Superior en Nutrición Infantil; Diplomado en Docencia Superior; Especialista en Pediatría; Doctor en Medicina y Cirugía; Universidad de Guayaquil, Guayaquil, Ecuador.

Iv. Master en Gerencia Educativa, Especialista en Gestión de Procesos Educativos, Diplomado en Docencia Superior; Universidad de Guayaquil, Guayaquil, Ecuador.
} 
Filipo P. Montece-López; Marlene I. Saltos-Calvache; Carlota M. Palma-Estrada; Julio De la Torre-Chávez

\section{Resumen}

En neonatología la principal causa del paso del virus VIH de la madre al neonato, se da mediante la trasmisión vertical durante el parto vaginal de acuerdo a la situación de infección materna. Se estudió en esta investigación a los neonatos expuestos por madres gestantes seropositivas en el Hospital Mariana de Jesús; se evidencia la importancia de los controles prenatales, diagnóstico oportuno, profilaxis previa, y el esquema del tratamiento idóneo, con el objetivo de disminuir las tasas de transmisión vertical que continúan siendo prevalencia a nivel mundial del VIH. Se comprobó que existe la correlación entre la situación de la madre gestante y la vitalidad del recién nacido a los cinco minutos de vida; se demuestra que el causal es la gravedad del VIH en la madre, sin diagnóstico oportuno, ni profilaxis previa, por ello la probabilidad que el Test de APGAR del neonato al nacer sea menos a siete puntos es mayor, a diferencia de las madres que han recibido tratamiento oportuno y controles para disminuir los índices de morbimortalidad de esta enfermedad y los riesgos neurológicos del bebe.

Palabras claves: VIH; neonatos; transmisión; diagnóstico; profilaxis. 


\section{Summary}

In the case of neonates the main risk of HIV acquisition is due to vertical transmission during the delivery according to the situation of maternal infection in the Hospital Mariana of Jesus. The objective of our study was to assess the importance of the prenatal care; timely diagnosis, preexposure prophylaxis, and the impact of this factor in the rate of vertical transmission, with the aim of reducing rates vertical transmission remain prevalent of HIV worldwide. The main finding of our result was correlation between the situation of the pregnant mother and vitality of the newborn within five minutes of life, it is shown that the causal is the seriousness of HIV in the mother, without timely diagnosis, or pre-exposure prophylaxis, the probability higher is the APGAR less seven points at birth, unlike mothers who have received timely treatment and controls to reduce morbidity and mortality rates of this disease and neurological risks of baby.

Key words: HIV; neonates; transmission; diagnostic; prophylaxis. 
Filipo P. Montece-López; Marlene I. Saltos-Calvache; Carlota M. Palma-Estrada; Julio De la Torre-Chávez

\section{Resumo}

No caso dos neonatos, o principal risco de aquisição do HIV deve-se à transmissão vertical durante o parto de acordo com a situação de infecção materna no Hospital Mariana de Jesus. O objetivo do nosso estudo foi avaliar a importância do atendimento pré-natal; Diagnóstico atempado, profilaxia pré-exposição e o impacto desse fator na taxa de transmissão vertical, com o objetivo de reduzir as taxas, a transmissão vertical continua a prevalecer do HIV em todo o mundo. O principal achado do nosso resultado foi a correlação entre a situação da mãe grávida e a vitalidade do recém nascido dentro de cinco minutos da vida, mostra-se que a causalidade é a gravidade do HIV na mãe, sem diagnóstico oportuno ou profilaxia pré-exposição, A probabilidade maior é a APGAR menos sete pontos ao nascer, ao contrário das mães que receberam tratamento e controles atempados para reduzir as taxas de morbidade e mortalidade desta doença e os riscos neurológicos do bebê.

Palavras-chave: HIV; neonatos; transmissão; diagnóstico; profilaxia. 


\section{Introduccion.}

A nivel mundial el Virus Inmunodeficiencia Humana (VIH) es una problemática en salud pública, al reportar 36,9 millones de vidas infectadas hasta el año 2014, sin dejar de considerar que 1,2 millones fallecieron debido a este virus, según datos de la Organización Mundial de la Salud (OMS, 2014).

La infección por el VIH se diagnostica mediante el análisis de sangre en los que se detecta la presencia o ausencia de anticuerpos contra el virus. Es importante mencionar que no existe una cura actual para la infección, los pacientes pueden mantener controlado el virus y llevar una calidad de vida y productiva siguiendo un tratamiento eficaz con fármacos antirretrovírales, a mediados del 2015 había 15,8 millones de pacientes infectados que recibían terapia antirretrovírica en todo el mundo.

Se pone de manifiesto que la mayor parte de mujeres gestantes no se efectúan el examen de VIH, son quienes trasmiten el virus al neonato durante el parto vaginal o mediante la lactancia materna, se denomina transmisión vertical, es la causa principal por lo que 260.000 nacen cada año con virus VIH por esta vía de contacto. En el Ecuador hasta el 2014 se han reportado 33.000 personas viven con el virus VIH, presentando una disminución en comparación con el año 2013 de 37.000 infectados según datos de ONUSIDA.

Según recomendaciones de Organización Panamericana de la Salud (OPS) en el año 2013, se comenzó aplicar tratamiento antirretroviral en etapas tempranas de la enfermedad, y de manera prioritaria a mujeres gestantes con el fin de evitar la transmisión vertical, eliminar el SIDA, aumentar la expectativa y calidad de vida en los ecuatorianos. De acuerdo a las estrategias 
Filipo P. Montece-López; Marlene I. Saltos-Calvache; Carlota M. Palma-Estrada; Julio De la Torre-Chávez

establecidas en el Plan del Buen Vivir, el Ecuador garantiza a la población salud pública accesible gratuita y con atención integral, con enfoque de género, comunitario e intercultural.

La ciudad de Guayaquil, es la más poblada de la república del Ecuador. Por la influencia que tiene a nivel regional en el ámbito comercial, político, financiero, cultural, tecnológico y en salud. Cuenta con una densidad de población de 2`531.223 habitantes urbanos en el 2013 (INEN, 2013). Está ubicado el Hospital Especializado Mariana de Jesús, donde se obtiene 8.042 nacimientos al año, según datos estadística 2015.

De los cuales 247 niños constan en el Programa de VIH, con el que cuenta esta institución para brinda el apoyo, control y vigilancia a las madres infectadas con el virus, desde la primera consulta, en la semana 36 de gestación y en el parto o cuando son de captación tardía son integradas en el programa.

Entre los nacimientos obtenidos en el año 2015, en el Hospital Especializado Mariana de Jesús se presentó 3 casos de neonatos con transmisión vertical, de madres infectadas sin control gestacional previos ni profilaxis.

\section{Importancia del Problema}

El presente proyecto nace de la necesidad de correlacionar la situación materna gestacional y la vitalidad del recién nacido al minuto y cinco minutos de vida determinando el desarrollo neurológico, mediante análisis comparativo, cohorte retrospectivo y monocéntrico, que permitirá obtener variables de resultados que ayudarán a estructurar estrategias de prevención y control del binomio madre-hijo, con el fin de disminuir los índices de morbimortalidad y erradicar los nuevos casos de infección por VIH en niños, de acuerdo a los compromisos realizados como Plan Mundial 
establecido en la reunión de las Naciones Unidas de junio del 2011. En este trabajo de investigación se plantea la hipótesis que a mayor gravedad de la situación materna de TARGA VIH/SIDA, menor puntuación en la escala de vitalidad del recién nacido mediante el Test de Puntuación APGAR al minuto y cinco minutos de vida, donde se determina el desarrollo neurológico del neonato.

\section{Metodología.}

Investigación se ha utilizado datos estadísticos de neonatos expuesto de madres seropositivas entre el período 2013 al 2015, mediante estudio analítico comparativo, cohorte retrospectivo y monocéntrico.

\section{Resultados.}

Datos estadísticos utilizados en el período de estudio desde el año 2013 al 2015 de madres en situación de TARGA VIH/SIDA, han expuesto al recién nacido ante el virus, de los cuales han sido 385 y se determinará la escala de vitalidad mediante APGAR al minuto y cinco minuto de vida. Se observa en la tabla1 la mayor frecuencia porcentual se ubicó en Edad Gestacional de 37 a 40 semanas de gestación, con un 71\%, que correspondió a 274 pacientes en un período trianual. El 75\% de los neonatos estudiados (de 385 fueron $290 \mathrm{RN}$ ), correspondieron a niños adecuados para la Edad Gestacional. Solo el $1 \%$ corresponde a grande para la Edad Gestacional tal como demuestra la ilustración 2.

El 99\% de los RN se obtuvieron vía cesárea, debido a su situación materna con TARGA VIH, sin embargo se observaron un total de 5 casos en período trianual que nacieron por parto vaginal, porque llegaron con dilatación completa y trabajo de parto, y no pudo revertirse a cesárea, causal por lo que se obtuvieron RNPT. El 58\% de la muestra pertenecen al género masculino (225 
Filipo P. Montece-López; Marlene I. Saltos-Calvache; Carlota M. Palma-Estrada; Julio De la Torre-Chávez

pacientes), y el $42 \%$ al femenino de neonatos obtenidos de madres con TARGA VIH, tal como demuestra la ilustración 4 .

El $56 \%$ de los neonatos corresponde a 215 pacientes y sus familias refirieron una situación económica en nivel 1 (con ingresos variables inferiores a un salario básico), seguido de un 19\% en situación 3 (ingresos variables mayores a un salario básico), demuestra la situación económica familiar tal como lo demuestra la ilustración 5.

Se evidencia que únicamente el $98 \%$ de las pacientes tuvieron 5 o más controles prenatales durante su embarazo, pese a su situación HIV. En el estudio se evidencia que el $60 \%$ de las gestantes (231 pacientes) en el período trianual 2013-2015 se encontraban en situación 1, seguido de un 26\% (99 pacientes) en situación 2. Únicamente un 14\% en situación 3 (55 pacientes). No hubo casos en situación 4, sin embargo el $98 \%$ de las gestantes corresponde a 378 pacientes, si recibieron tratamiento antirretroviral previo a la atención del parto, a diferencia del $2 \%$ que no recibieron tratamiento, tal como lo demuestra la ilustración 8. El 99\% de las gestantes si recibieron profilaxis materna durante el parto, correspondiente a 383 pacientes. El 1\% en el año 2013 corresponde a la discordancia.

Entre los esquemas antirretrovirales el 58\% de las gestantes recibieron esquema con efv-emttenofovir, correspondiente a 223 pacientes, seguido de $26 \%$ con esquema de lopinavir - ritonavir (99 gestantes), y 14\% (55 pacientes), con esquema de ralt- (emt+tenof)- ritonavir. Considerando los factores sociales familiares de nuestro entorno, el 93\% (358 pacientes), tuvieron adherencia adecuada al tratamiento, mientras que un $7 \%$ no lo tuvo.

En el primer mes de vida se les realizo el Test de ELISA, donde se obtuvieron 383 neonatos que corresponde al $99 \%$ siendo seropositivos en la muestra, sin embargo se observa el 1\% son 
seronegativos. El 92,7\% de los neonatos, corresponde a 357 pacientes tuvieron un APGAR de 7 a 10 puntos a los 5 minutos de vida, seguido de un $7 \%$ de pacientes que corresponde a 27 neonatos, y tan solo un 0,3\% de la muestra es decir 1 paciente, obtuvo APGAR de 1 a 3 puntos considerado asfixia perinatal severa.

\section{Discusión.}

Según los resultados obtenidos en este estudio de investigación realizado en el Hospital Mariana de Jesús, el número de variables ante los objetivos preestablecidos, determina que las madres gestantes con TARGA VIH, en un amplio rango reciben control previos gestacional y profilaxis para evitar la transmisión vertical durante el trabajo de parto y tratamiento, se consigue alcanzar tasas inferiores al 2\% de transmisión vertical. La tasa encontrada en el hospital fue del $1 \%$ de trasmisión vertical, por debajo de la tasa bibliográfica.

El APGAR a los 5 minutos de vida es útil para la evaluación rápida y efectiva del recién nacido mediante la valoración de 5 parámetros: frecuencia cardiaca, frecuencia respiratoria, tono muscular, reflejos y color de la piel, se valora en dos tiempos, según Virginia Apgar en 1953.

El análisis y reporte de este estudio de neonatos expuestos al VIH de madres seropositivas mediante la transmisión vertical, suelen nacer a término por vía cesárea con longitud acorde a su edad gestacional, aunque es evidente observar neonatos de bajo peso y con la presencia de síntomas de abstinencia. (Paganini, 2007)

El estudio realizado sobre VIH a nivel internacional demuestra que la trasmisión vertical oscila entre $15 \%$ - 45\%, pero es posible prevenir con la administración de antirretrovirales, según la Organización Mundial de la Salud en el 2015. En el hospital se evidencia gestantes de madres con 
Filipo P. Montece-López; Marlene I. Saltos-Calvache; Carlota M. Palma-Estrada; Julio De la Torre-Chávez

situación de estadio 3, los neonatos han presentado APGAR menor a 7 a los 5 minutos de vida.

(Fonseca \& Prieto, 2005)

En las bibliografías revisadas las manifestaciones clínicas de manera precoz en neonatos prematuros y de término, por trasmisión vertical entre ellas lesión cerebral hipóxico isquémica, encefalopatía, y otras infecciones oportunistas es más evidente la presencia de signos inmunológicos y virales.

La encefalopatía hipóxico isquémica es una de la principales causas de morbimortalidad en neonatos prematuros o de término, oscila entre 0,5-1/1.000 nacidos vivos, tiene una relación con la incidencia de asfixia perinatal que oscila entre 3-5/1.000 nacidos vivos. Es detectable con el test de APGAR que nos determina la viabilidad de recién nacido a los 5 minutos de vida. (Cabañas \& Valverde, 2011).

\section{Conclusión.}

Del total de neonatos expuestos (385 neonatos), atendidos en el Hospital Marianas de Jesús, hijos de gestantes expuestas al VIH, en el período trianual 2013-2015, se observó que el 75\% fueron RN a término, es decir adecuados para la edad gestacional, 56\% con una situación Socio Económica Familiar de 1 (ingresos variables menores a un sueldo básico), 27\% de las gestantes no tuvieron controles prenatales adecuados, lo cual continúa siendo un problema socio-cultural, pese a su situación VIH. El $60 \%$ se encontraban en situación 1, con un $16 \%$ de pacientes sin tratamiento antirretroviral. El 1\% no recibió profilaxis materna por causa de la discordancia. El 58\% recibieron esquema ARV de efv + emt + tenofovir. De ellas, un 7\% no tuvo adherencia adecuada al tratamiento. 
En relación a la problemática expuesta en el presente estudio, la correlación entre APGAR menor a 7 puntos a los 5 minutos de vida y situación materna del HIV de 3 o más, dio una razón de productos cruzados de 2,20, estadísticamente significativa. Por tanto se valida la hipótesis verdadera.

Con el antecedente antes descrito se concluye que, se requiere fomentar acciones en Salud Pública para mejorar la captación de pacientes expuestas, asegurar un tratamiento ARV oportuno, fomentar sus controles prenatales de forma exhaustiva, y mejorar la adherencia al tratamiento. De este modo se puede reducir el riesgo estadístico de APGAR bajo al nacer, se encuentra condicionado a la situación materna HIV de mayor gravedad.

\section{Bibliografía.}

Cabañas, F., \& Valverde, E. (2011). Encefalopatía Hipóxica en el Recién Nacido. En A. Sola, Cuidados Neonatales. Descubriendo la vida de un recién nacido enfermo (Vol. II). Argentina: Edimed.

Paganini, H. R. (2007). Infectología Pediátrica (Primera ed.).

Fonseca, C. E., \& Prieto, F. E. (2005). Manejo de la Infección Materna VIH y del Recién Nacido Expuesto. Revista Colombiana de Obstetricia y Ginecología, 56. 
Filipo P. Montece-López; Marlene I. Saltos-Calvache; Carlota M. Palma-Estrada; Julio De la Torre-Chávez

\section{nexos}

Tabla 1. Edad Gestacional

\begin{tabular}{|l|r|r|r|r|c|}
\hline 1. Edad Gestacional & $\mathbf{2 0 1 3}$ & $\mathbf{2 0 1 4}$ & $\mathbf{2 0 1 5}$ & Total & $\%$ \\
\hline menor a 33 & 10 & 12 & 16 & 38 & $10 \%$ \\
\hline 34 a 36 & 14 & 21 & 27 & 62 & $16 \%$ \\
\hline 37 a 40 & 59 & 89 & 126 & 274 & $71 \%$ \\
\hline mayor de 40 & $\mathbf{3}$ & $\mathbf{2}$ & $\mathbf{6}$ & $\mathbf{1 1}$ & $\mathbf{3 \%}$ \\
\hline Total & $\mathbf{8 6}$ & $\mathbf{1 2 4}$ & $\mathbf{1 7 5}$ & $\mathbf{3 8 5}$ & $\mathbf{1 0 0 \%}$ \\
\hline
\end{tabular}

Tabla 2. Talla para la Edad Gestacional

\begin{tabular}{|l|r|r|r|r|r|}
\hline 2. Talla / EG & 2013 & $\mathbf{2 0 1 4}$ & $\mathbf{2 0 1 5}$ & Total & \% \\
\hline AEG & 63 & 93 & 134 & 290 & $75 \%$ \\
\hline PEG & 22 & 30 & 39 & 91 & $24 \%$ \\
\hline GEG & 1 & 1 & 2 & 4 & $1 \%$ \\
\hline Total & $\mathbf{8 6}$ & 124 & 175 & 385 & $100 \%$ \\
\hline
\end{tabular}

Tabla 3. Vía de Atención del Parto

\begin{tabular}{|l|r|r|r|r|c|}
\hline 3. Vía de Atención del Parto & 2013 & 2014 & 2015 & Total & $\%$ \\
\hline Parto Vaginal & 1 & 2 & 2 & 5 & $1 \%$ \\
\hline Cesárea & 85 & 122 & 173 & 380 & $99 \%$ \\
\hline Total & 86 & 124 & 175 & 385 & $100 \%$ \\
\hline
\end{tabular}

Tabla 4. Género de Neonatos Expuestos

\begin{tabular}{|l|r|r|r|r|c|}
\hline 4. Género de la muestra & 2013 & 2014 & 2015 & Total & $\%$ \\
\hline Femenino & 40 & 46 & 74 & 160 & $42 \%$ \\
\hline Masculino & 46 & 78 & 101 & 225 & $58 \%$ \\
\hline Total & 86 & 124 & 175 & 385 & $100 \%$ \\
\hline
\end{tabular}

Tabla 5. Situación Socio Económico Familiar

\begin{tabular}{|l|rrrrr|}
\hline 5. Situación Socio Económica Familiar & $\mathbf{2 0 1 3}$ & 2014 & 2015 & \multicolumn{1}{c|}{ Total } & $\%$ \\
\hline 1 (variables menor a SB) & 43 & $7 €$ & 96 & $21 j$ & $56 \%$ \\
\hline 2 (fijos menor a SB) & 16 & 15 & 27 & 58 & $15 \%$ \\
\hline 3 (variables mayor a SB) & 18 & 22 & 33 & 73 & $19 \%$ \\
\hline 4 (fijos mayor a SB) & 9 & 11 & 19 & 39 & $10 \%$ \\
\hline
\end{tabular}

Tabla 6. Controles Prenatales

\begin{tabular}{|c|c|c|c|c|c|}
\hline 6. CPN & 2013 & 2014 & 2015 & Total & $\%$ \\
\hline Si & 85 & 122 & 171 & 378 & $98 \%$ \\
\hline No & 1 & 2 & 4 & 7 & $2 \%$ \\
\hline Total & 86 & 124 & 175 & 385 & $100 \%$ \\
\hline
\end{tabular}


Tabla 7. Situación VIH Materna

\begin{tabular}{|l|rrrrc|}
\hline 7. Situación VIH Materna & $\mathbf{2 0 1 3}$ & $\mathbf{2 0 1 4}$ & $\mathbf{2 0 1 5}$ & Total & $\%$ \\
\hline Sit. 1 & 49 & 72 & 110 & 23 & $60 \%$ \\
\hline Sit. 2 & 25 & 32 & 42 & 99 & $26 \%$ \\
\hline Sit. 3 & 12 & 20 & 23 & 55 & $14 \%$ \\
\hline Total & $\mathbf{8 6}$ & $\mathbf{1 2 4}$ & $\mathbf{1 7 5}$ & $\mathbf{3 8}$ & $\mathbf{1 0 0 \%}$ \\
\hline
\end{tabular}

Tabla 8. Tratamiento Antirretroviral Previo Materno

\begin{tabular}{|l|r|r|r|r|r|r|}
\hline 8. Tratamiento ARV Previo & 2013 & $\mathbf{2 0 1 4}$ & $\mathbf{2 0 1 5}$ & Total & $\%$ \\
\hline Si & 81 & 122 & 171 & 378 & $98 \%$ \\
\hline No & 1 & 2 & 4 & 7 & $2 \%$ \\
\hline Total & 86 & 124 & 175 & 385 & $100 \%$ \\
\hline
\end{tabular}

Tabla 9. Profilaxis Materna

\begin{tabular}{|l|r|r|r|r|r|r|}
\hline 9. Profilaxis materna & 2013 & 2014 & $\mathbf{2 0 1 5}$ & Total & \% \\
\hline Si & 84 & 124 & 175 & 383 & $99 \%$ \\
\hline No & 2 & 0 & 0 & 2 & $1 \%$ \\
\hline
\end{tabular}

Tabla 10. Esquemas Antirretrovirales

\begin{tabular}{|l|rrrr|c|}
\hline 10. Esquemas ARV & $\mathbf{2 0 1 3}$ & 2014 & 2015 & Total & $\%$ \\
\hline Ninguno & 2 & 1 & 5 & 8 & $2 \%$ \\
\hline efv + emt + tenofovir & 47 & 71 & 105 & 22 & $58 \%$ \\
\hline lopinavir + ritonavir & 25 & 32 & 42 & 99 & $26 \%$ \\
\hline ralt + (emt+tenof) + rito & 12 & 20 & 23 & 55 & $\mathbf{1 4 \%}$ \\
\hline Total & 86 & 124 & 175 & $38 ;$ & $100 \%$ \\
\hline
\end{tabular}

Tabla 11. Adherencia al Tratamiento Antirretroviral

\begin{tabular}{|l|r|r|r|r|c|}
\hline 11. Adherencia al tratamiento ARV & 2013 & 2014 & 2015 & Total & $\%$ \\
\hline Si & 80 & 115 & 163 & 358 & $93 \%$ \\
\hline No & 6 & 9 & 12 & 27 & $7 \%$ \\
\hline Total & 86 & 124 & 175 & 385 & $100 \%$ \\
\hline
\end{tabular}

Fuente: Filipo Montecè y Marlene Saltos, Ecuador, 2017

Tabla 12. Situación VIH del Recién Nacido

\begin{tabular}{|l|rrrr|c|}
\hline 12. Situación VIH- RN & $\mathbf{2 0 1 3}$ & $\mathbf{2 0 1 4}$ & $\mathbf{2 0 1 5}$ & Total & $\%$ \\
\hline Seropositivo & 84 & 124 & 175 & 38 & $99 \%$ \\
\hline Seronegativo & 2 & 0 & 0 & 2 & $1 \%$ \\
\hline & 86 & 124 & 175 & 38 & $100 \%$ \\
\hline
\end{tabular}


Filipo P. Montece-López; Marlene I. Saltos-Calvache; Carlota M. Palma-Estrada; Julio De la Torre-Chávez

Tabla 13. APGAR 5 minutos de vida RN

\begin{tabular}{|c|c|c|c|c|c|}
\hline 13. APGAR 5 $\mathbf{~ m i n}$ & $\mathbf{2 0 1 3}$ & $\mathbf{2 0 1 4}$ & $\mathbf{2 0 1 5}$ & Total & \% \\
\hline $\mathbf{1 - 3}$ & 0 & 0 & 1 & 1 & $0,3 \%$ \\
\hline $\mathbf{4}-\mathbf{6}$ & 9 & 8 & 10 & 27 & $7 \%$ \\
\hline $\mathbf{7 - 1 0}$ & 77 & 116 & 164 & 357 & $92,7 \%$ \\
\hline Total & 86 & 124 & 175 & 385 & $100 \%$ \\
\hline
\end{tabular}

